\title{
Different effects of dark treatment on pigment composition and photosystem I and II activities in intact cotyledons and primary leaves of Cucurbita pepo (zucchini)
}

\author{
Kiril Mishev · Detelin Stefanov • Kalina Ananieva • \\ Chavdar Slavov · Evguéni D. Ananiev
}

Received: 29 April 2008/ Accepted: 4 December 2008/Published online: 27 December 2008

(C) Springer Science+Business Media B.V. 2008

\begin{abstract}
Darkness mediates different senescencerelated responses depending on the targeting of dark treatment (whole plants or individual leaves) and the organs that perceive the signal (leaves or cotyledons). In this study, we presented data on the differential effects of 2-day dark treatment on progression of senescence in cotyledons and primary leaves of 14day-old plants of Cucurbita pepo L. (zucchini). The lack of changes in chlorophyll $a$, chlorophyll $b$ and carotenoid contents as well as in the PSI activity measured by the far-red induced alterations in the $\mathrm{P}_{700}$ oxidation levels and the quantum yield of electron transport from $Q_{\mathrm{A}}{ }^{-}$to PSI end acceptors $\left(\varphi_{\mathrm{R}_{0}}\right)$ indicated higher resistance of cotyledons to the applied dark stress compared to the primary leaves. In contrast to cotyledons, PSI activity in the primary
\end{abstract}

K. Mishev · D. Stefanov · K. Ananieva

Institute of Plant Physiology, Bulgarian Academy of Sciences, Acad. G. Bonchev str., bl. 21, 1113 Sofia, Bulgaria

Present Address:

C. Slavov

Max-Plank-Institute für Bioanorganische Chemie,

Stiftstr. 34-36, 45413 Mulheim an der Ruhr, Germany

E. D. Ananiev $(\square)$

Department of Plant Physiology, Faculty of Biology,

St. Kl. Ohridski University of Sofia,

8 Dragan Tsankov bld., 1164 Sofia, Bulgaria

e-mail: ananiev@bio21.bas.bg leaves was significantly inhibited. Concerning the activity of PSII analyzed by the changes in the JIPtest parameters (the maximal efficiency of PSII photochemistry, $\varphi_{\mathrm{P}_{0}}$; the performance index, $\mathrm{PI}_{\mathrm{ABS}}$; the efficiency of $Q_{\mathrm{A}}{ }^{-}$reoxidation, $\psi_{0}$ and the effective dissipation per reaction center, $\mathrm{DI}_{0} / \mathrm{RC}$ ), no differences were observed between cotyledons and primary leaves, thus suggesting that PSI activity in the true leaves was more susceptible to the applied dark stress. The transfer of the darkened plants to normal light regime resulted in delayed senescence in cotyledons which was in contrast to results on Arabidopsis, thus implying the existence of specific mechanisms of cotyledon senescence in different monocarpic plants.

Keywords Dark-induced senescence and recovery . Cotyledons and primary leaves .

PSII and PSI activity · JIP-test

$\begin{array}{ll}\text { Abbreviations } \\ \text { PSA } & \text { Photosynthetic apparatus } \\ \text { PSI } & \text { Photosystem I } \\ \text { PSII } & \text { Photosystem II } \\ \text { RC } & \text { PSII reaction center } \\ \text { Chl } & \text { Chlorophyll } \\ \text { Car } & \text { Carotenoids } \\ \text { ABS } & \text { Absorption flux } \\ \mathrm{TR}_{0} & \text { Trapped energy flux (at } t=0) \\ \mathrm{ET}_{0} & \text { Electron transport flux }(\text { at } t=0) \\ \mathrm{DI}_{0} & \text { Dissipated energy flux }(\text { at } t=0)\end{array}$


$\mathrm{RE}_{0}$ The flux referring to the reduction of end acceptors at the PSI electron acceptor side (at $t=0$ )

DP Plants darkened for 2 days

RDP Darkened plants recovered for 9 days in normal light regime

PI Performance index

\section{Introduction}

Plant senescence comprises a series of genetically controlled deteriorative changes and is considered the final phase of plant/organ development that culminates in death (Nooden 1988; Smart 1994). One of the earliest events of leaf senescence is the decline of the photosynthetic function and the loss of chloroplast structures (Nooden et al. 1997; Krupinska and Humbeck 2004). Chloroplast senescence is a complex process of different senescence-associated events including degradation of pigments, proteins, membrane lipids and nucleic acids resulting in complete chloroplast breakdown (Krupinska and Humbeck 2004). During chloroplast senescence, a decrease in photosynthetic function has been manifested by intensive degradation of ribulose-1,5-bisphosphate carboxylase (Rubisco) and other stromal enzymes (Grover 1993). In comparison with the stromal proteins, the degradation of the thylakoid proteins of PSII, PSI and the light-harvesting complexes (LHCs) of both photosystems is accelerated at later stages of leaf senescence (Humbeck and Krupinska 2003).

Different environmental stress factors, such as extreme temperatures, drought, nutrient deficiency, shading or total darkness can induce senescence at any stage of plant life cycle (Thomas and Stoddart 1980). The ability of darkness to accelerate senescence has been studied in detached leaves (Thimann 1980; Oh et al. 1996) or intact plants (Biswal and Biswal 1984; $\mathrm{Lu}$ and Zhang 1998). However, it has been demonstrated that in Arabidopsis leaf senescence is not induced, but in fact is inhibited when a whole plant is darkened whereas in contrast, senescence is strongly accelerated in individually darkened leaves (Weaver and Amasino 2001). Thus, darkness can induce two different senescence-related responses summarized in a model proposed by Weaver and Amasino (2001). According to this model, darkness can provoke either promotion of senescence when applied locally, at the cell, tissue and/or organ level or repression of senescence when it is expressed at the level of the entire plant. Recently reported data have indicated that while photosynthetic capacity of whole darkened Arabidopsis plants remained unchanged, the mitochondrial respiration decreased (Keech et al. 2007). In contrast, in individually darkened leaves a rapid decline in photosynthetic activity was found while maintaining high mitochondrial respiration. The changes provoked by the two treatments were accompanied with an unequal degradation of chloroplasts and mitochondria in the senescing leaves (Keech et al. 2007).

Most of the data on dark-induced senescence were obtained with leaves, but much less is known about the response of cotyledons to darkness. Cotyledons represent specific reserve organs with the main physiological function to ensure the growth of the seedlings at the early stages of germination. Moreover, epigeal cotyledons are the first photosynthesizing organs in plant ontogenesis which undergo a gradual transition from mixotrophic to fully autotrophic metabolism (Kutik et al. 1998). It has been reported that chloroplast senescence in the cotyledons of Brassica napus progresses in a sequential manner including a sharp decline in PSII activity accompanied by marked structural changes in the thylakoids (Ghosh et al. 2001). In senescing Cucumis sativus cotyledons an age-dependent migration of the LHCII complex and its association with PSI leading possibly to destacking of grana structure was found (Prakash et al. 2003). Our recent results on natural senescence of Cucurbita pepo (zucchini) cotyledons have shown that the decline in the net photosynthetic rate estimated by $\mathrm{CO}_{2}$ uptake started earlier in ontogenesis compared with the downregulation of PSII electron transport (Ananieva et al. 2008a). Thus, during earlier stages of natural senescence, the existence of protective mechanisms maintaining higher efficiency of the photochemical electron transport reactions compared with the dark reactions of the Calvin cycle could be suggested.

The effect of darkness on cotyledon senescence was studied after transfer of intact 7-day-old Cucurbita pepo (zucchini) seedlings to darkness for $24 \mathrm{~h}$ (Ananieva et al. 2007). The results showed no effect on both the maximal PSII quantum yield estimated by the $F_{\mathrm{V}} / F_{\mathrm{M}}$ ratio and the rate of $\mathrm{CO}_{2}$ uptake. On the other hand, dark treatment caused a decrease in total 
chloroplast RNA synthesis and in the content of biologically active cytokinins. Prolongation of the dark treatment of zucchini seedlings (5 days) resulted in promotion of cotyledon senescence as judged by the progressive reduction in chlorophyll content and deterioration of chloroplast ultrastructure (Ananieva et al. 2004). These changes correlated with a reduction in the content of bioactive CKs which are the major senescence-inhibiting hormones (van Staden et al. 1988; Kim et al. 2006). In contrast to developmental cotyledon senescence, the storage cytokinin $O$-glucosides were decreased only in the cotyledons of whole darkened plants, suggesting different metabolic regulation of endogenous CK levels during natural and dark-induced senescence. Besides, involvement of cytokinin oxidase/dehydrogenase in cytokinin signaling during senescence has been recently demonstrated in individually darkened cotyledons (Ananieva et al. 2008b).

Although senescence of cotyledons is not fundamentally different from leaf senescence, organ-specific differences between cotyledons and differentiated leaves with respect to their photosynthetic activity during natural senescence have been reported (La Rocca et al. 1996). It was shown that in contrast to differentiated leaves cotyledon senescence in Arabidopsis was induced when whole plants were placed in darkness thus indicating that dark-induced senescence can be organ-specific (Weaver and Amasino 2001). On the other hand, it must be also taken into account that senescence in Arabidopsis is different from that of other monocarpic plants in that the leaf longevity is not controlled by the developing reproductive structures (Lim et al. 2007). Thus, the senescence mechanisms found in Arabidopsis might not be the same as in other monocarpic plants, such as zucchini. Furthermore, the differential response of the two leaf organs to darkness has been insufficiently studied.

The aim of the present work was to study the organspecific response of both cotyledons and primary leaves of Cucurbita pepo L. (zucchini) after transfer of whole plants to darkness by following the changes in pigment composition as well as PSII and PSI activities evaluated by chlorophyll $a$ fluorescence induction and far-red induced changes in $A_{830}$ leaf absorbance, respectively. The ability of the photosynthetic activity of cotyledons and leaves to recover after returning to normal light regime was also investigated.

\section{Materials and methods}

Growth conditions and treatments

Seeds of Cucurbita pepo L. (zucchini) were germinated on moistened filter paper in darkness at $28^{\circ} \mathrm{C}$ for $96 \mathrm{~h}$. The 4-day-old etiolated seedlings were grown further on a nutrient solution (Yamagishi and Yamamoto 1994) in a growth chamber at a photon flux density of $100 \mu \mathrm{mol} \mathrm{m}{ }^{-2} \mathrm{~s}^{-1}, 26 \pm 2^{\circ} \mathrm{C}$, relative humidity of $60 \%$ and a $12 / 12 \mathrm{~h}$ day/night cycle. Under these conditions cotyledons of the control plants reached their maximum photosynthetic activity on days 7-8 after the onset of germination (Mishev et al. 2005) while the maximum of the first differentiated leaf was registered on days 15-16 (data not shown). Cotyledons started to yellow earlier (22-25 day) compared with the primary leaves (25-28 day). Dark treatment was applied for 2 days at the age of 14 days when the seedlings had fully expanded primary leaves with maximal photosynthetic activity and the pair of cotyledons showed a slight decrease of the main photosynthetic parameters (Mishev et al. 2005). After the dark treatment plants were returned to normal light regime. Samples were collected 5 and 9 days after the plants had been returned to the light.

Chlorophyll and carotenoid contents determination

Chl and Car were extracted in $80 \%$ (v/v) acetone as described by Arnon (1949) and their contents were estimated using the extinction coefficients of McKinney (1941). Results represent the mean data of 4 independent experiments.

\section{Fluorescence measurements}

Chl $a$ fluorescence transients of dark-adapted zucchini cotyledons and primary leaves were measured at $25^{\circ} \mathrm{C}$ and recorded for $5 \mathrm{~s}$ using a Plant Efficiency Analyser (PEA; Hansatech Ltd, King's Lynn, Norfolk, UK) after 15 min dark adaptation. The fluorescence transients were induced by continuous red light $(650 \mathrm{~nm}$ peak wavelength and intensity of 3,000 $\operatorname{molm}^{-2} \mathrm{~s}^{-1}$ PFD) provided by PEA through an array of six lightemitting diodes. The following data from the original 
measurements were used: the maximal fluorescence intensity, $F_{\mathrm{P}}$, equal to $F_{\mathrm{M}}$ when all PSII reaction centers are closed; the fluorescence intensity at $50 \mu \mathrm{s}$ considered as the intensity $F_{0}$ when all reaction centers are open; the fluorescence intensities at $2 \mathrm{~ms}$ ( $\mathrm{J}$ step or $F_{\mathrm{J}}$ ) and $30 \mathrm{~ms}$ (I step or $F_{\mathrm{I}}$ ) describing intermediary changes in PSII redox state related to PQ pool filling up. The fluorescence transients were analyzed according to the JIP-test equations (Strasser et al. 2004). JIP-test parameters present the energy fluxes and their bifurcations, as well as the efficiencies/yields, which are defined as ratios of the energy fluxes. The following parameters were used: $\varphi_{\mathrm{P}_{0}}=\mathrm{TR}_{0} / \mathrm{ABS}=1-F_{0} / F_{\mathrm{M}}$ which is the maximum quantum yield of primary photochemistry; $\psi_{0}=\mathrm{ET}_{0} / \mathrm{TR}_{0}=\left(F_{\mathrm{M}}-F_{\mathrm{J}}\right) /\left(F_{\mathrm{M}}-F_{0}\right)$ which is the probability (at $t=0$ ) that a trapped exciton moves an electron into the electron transport chain beyond $Q_{\mathrm{A}}{ }^{-} ; \quad \varphi_{\mathrm{R}_{0}}=\mathrm{RE}_{0} / \mathrm{ABS}=1-F_{\mathrm{I}} / F_{\mathrm{M}}$ which is the quantum yield of electron transport from $Q_{\mathrm{A}}{ }^{-}$to PSI end electron transport acceptors (Tsimilli-Michael and Strasser 2008; Smit et al. 2008); $\mathrm{DI}_{0} / \mathrm{RC}=(\mathrm{ABS} /$ $\mathrm{RC})-\left(\mathrm{TR}_{0} / \mathrm{RC}\right)$ which is the dissipated energy flux per reaction center (at $t=0$ ); the photosynthetic Performance Index $\left(\mathrm{PI}_{\mathrm{ABS}}\right)$ which is a combined measure of the amount of photosynthetic reaction centers (RC/ABS), the maximal energy flux which reaches the PSII reaction center $\left(\mathrm{TR}_{0}\right)$, and the electron transport at the onset of illumination $\left(\mathrm{ET}_{0}\right)$ and is calculated as:

$$
\begin{aligned}
\mathrm{PI}_{\mathrm{ABS}}= & \frac{1-\left(F_{0} / F_{\mathrm{M}}\right)}{4\left(F_{300 \mu \mathrm{s}}-F_{0}\right) /\left(F_{\mathrm{J}}-F_{0}\right)} \times \frac{\left(F_{M}-F_{0}\right)}{F_{0}} \\
& \times \frac{\left(F_{M}-F_{J}\right)}{\left(F_{J}-F_{0}\right)}
\end{aligned}
$$

Plotted values were the means of 15 repetitions from three independent experiments.

\section{$\mathrm{P}_{700}$ redox state}

The redox state of $\mathrm{P}_{700}$ was investigated in vivo with a dual wavelength $(810 / 860 \mathrm{~nm})$ unit (Walz ED 700DW-E) attached to a PAM101E main control unit, thus detecting purer $\mathrm{P}_{700}$ related signals (Klughammer and Schreiber 1998). $\mathrm{P}_{700}$ was oxidized by irradiation with far-red light $\left(13.4 \mathrm{~W} \mathrm{~m}^{-2}\right)$ provided by a photodiode (FR-102, Walz, Effeltrich, Germany) that was controlled by the PAM 102 unit.
Statistical analysis

Comparison of means from at least three separate physiological experiments was done using the Student's $t$-test and the software package Statistica 5 .

\section{Results}

Pigment content

The maximal Chl content in zucchini cotyledons was registered on days 8-9 after the onset of germination followed by a gradual decline, the pigment degradation being more markedly accelerated at the final stages of ontogenesis (after day 20) (Fig. 1). Primary leaves emerged later in ontogenesis (7-8 days from the onset of germination) and the maximal Chl accumulation was registered on days 15-16, followed by a sharp decrease thereafter. As a rule, cotyledons became fully yellow and died on days 26-28 from the onset of germination while primary leaves died 4-5 days after the cotyledons, thus exhibiting a shorter life span.

A decrease in total Chl content (by 18\%) was registered in the primary leaves after 2-day darkening of whole plants (Fig. 1). On the other hand, no effect was observed in the cotyledons of the darkened

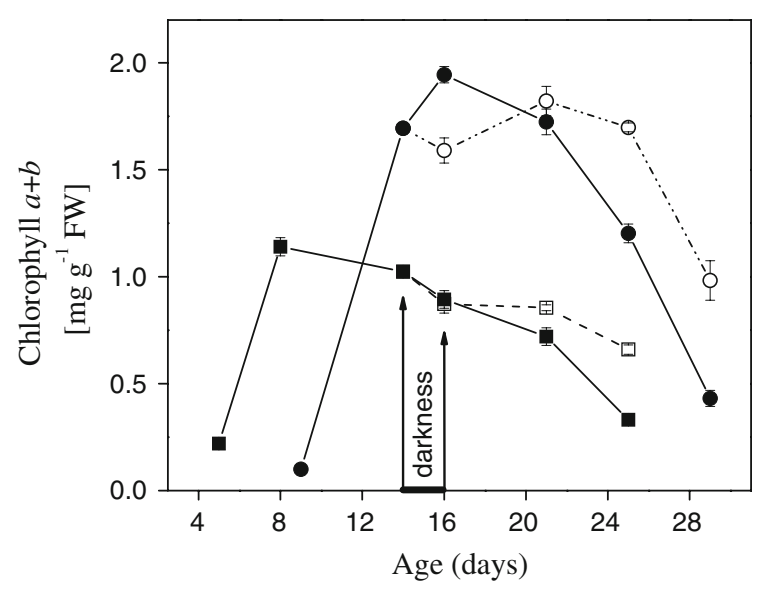

Fig. 1 Changes in total chlorophyll content in intact zucchini cotyledons and primary leaves during ontogenesis, after 2-day dark treatment of whole plants and during a recovery period. Results are expressed in $\mathrm{mg} \mathrm{g}^{-1}$ fresh weight $(\mathrm{FW})$. The values for the control cotyledons $(-\bullet)$ and primary leaves $(-\bullet)$, as well as for cotyledons $(-\square)$ and leaves ( $-\circ)$ of whole darkened plants are the means of four experiments 
plants. During the 9-day recovery period after returning plants to normal light regime $\mathrm{Chl}$ content in both cotyledons and primary leaves exceeded significantly that in controls (Fig. 1), thus indicating strongly delayed senescence.

The decline in total $\mathrm{Chl}$ content during natural senescence in both cotyledons and primary leaves was mainly due to $\mathrm{Chl} a$ (Table 1 ), thus leading to a decrease in the $\mathrm{Chl} a / b$ ratio. Two-day dark treatment did not change the $\mathrm{Chl} a / b$ ratio in both leaf organs. This was due to a similar reduction in both Chl $a$ and $\mathrm{Chl} b$ in the primary leaves (by $18 \%$ ) whereas in the cotyledons both $\mathrm{Chl} a$ and $\mathrm{Chl} b$ remained unchanged (Fig. 1). Nine days after returning the plants to normal light regime the $\mathrm{Chl} a / b$ ratio in the primary leaves was not significantly changed (Table 1). In contrast, the $\mathrm{Chl} a / b$ ratio in the cotyledons during the recovery period exceeded by $60 \%$ the values measured in senescing 25-day-old cotyledons due to the significantly higher content of Chl $a$ (2.4-fold) compared with the control. Besides, Chl $a$ content in the primary leaves after a 9-day recovery remained unchanged compared with the 2-day dark-treated leaves whereas $\mathrm{Chl} b$ content was increased reaching the values of the 16-day-old control leaves.

Total carotenoid (Car) content in the yellow cotyledons of 25-day-old plants was markedly reduced (by 45\%) compared with the 16-day-old cotyledons (Table 1). In the primary leaves, however, the observed decrease was slight for the same period of time. Two-day dark treatment of whole plants did not induce any significant changes in Car content in the cotyledons whereas a reduction by $23 \%$ was registered in the primary leaves (Table 1). A similar increase in total Car content (by 10\%) was observed in both cotyledons and primary leaves during the 9-day recovery period following reillumination, thus indicating delayed senescence.

JIP-test

A significant decline in the maximal efficiency of PSII photochemistry in the dark-adapted state $\varphi_{\mathrm{P}_{0}}$, equal to the most commonly used parameter in the literature $F_{\mathrm{V}} / F_{\mathrm{M}}$, was observed only at the later stages of ontogenesis (day 25) in yellow cotyledons as well as in the senescing primary leaves (Fig. 2a). On the other hand, the performance index (PI) which describes mainly PSII functional alterations (Strasser et al. 2004) was higher in the cotyledons compared with the primary leaves till day 16 followed by a gradual decrease in both leaf organs (Fig. 2b), being more pronounced in the cotyledons. It is well known that PI is a multiparametric expression including the

Table 1 Changes in Chl $a$, Chl $b$ and Car contents in zucchini cotyledons and primary leaves from control plants (C), 2 day darkened plants (DP) and plants recovered from the dark stress (RDP)

\begin{tabular}{lcccc}
\hline Variant & Chl $a$ & Chl $b$ & Car & Chl alb \\
\hline Cotyledons & & & & \\
C, 14 day & $0.716 \pm 0.02$ & $0.308 \pm 0.006$ & $0.141 \pm 0.004$ & 2.33 \\
C, 16 day & $0.618 \pm 0.02$ & $0.275 \pm 0.01$ & $0.134 \pm 0.006$ & 2.25 \\
DP, 16 day & $0.61 \pm 0.03$ & $0.26 \pm 0.01$ & $0.134 \pm 0.008$ & 2.35 \\
C, 25 day & $0.185 \pm 0.008$ & $0.144 \pm 0.01$ & $0.06 \pm 0.01$ & 1.29 \\
RDP, 25 day & $0.446 \pm 0.01$ & $0.215 \pm 0.007$ & $0.147 \pm 0.01$ & 2.07 \\
Primary leaf & & & $0.224 \pm 0.009$ & 2.51 \\
C, 14 day & $1.21 \pm 0.01$ & $0.483 \pm 0.007$ & $0.272 \pm 0.01$ & 2.41 \\
C, 16 day & $1.36 \pm 0.05$ & $0.564 \pm 0.002$ & $0.21 \pm 0.008$ & 2.39 \\
DP, 16 day & $1.12 \pm 0.05$ & $0.468 \pm 0.02$ & $0.25 \pm 0.01$ & 2.27 \\
C, 25 day & $0.834 \pm 0.02$ & $0.367 \pm 0.01$ & $0.231 \pm 0.01$ & 2.01 \\
RDP, 25 day & $1.13 \pm 0.003$ & $0.563 \pm 0.01$ & $0.119 \pm 0.002$ & 1.91 \\
C, 29 day & $0.283 \pm 0.025$ & $0.148 \pm 0.013$ & $0.163 \pm 0.014$ & 2.11 \\
RDP, 29 day & $0.667 \pm 0.062$ & $0.316 \pm 0.031$ & & \\
\hline
\end{tabular}

Results are expressed in $\mathrm{mg} \mathrm{g}^{-1}$ fresh weight $(\mathrm{FW})$. Mean values with the respective standard errors are based on four independent measurements 


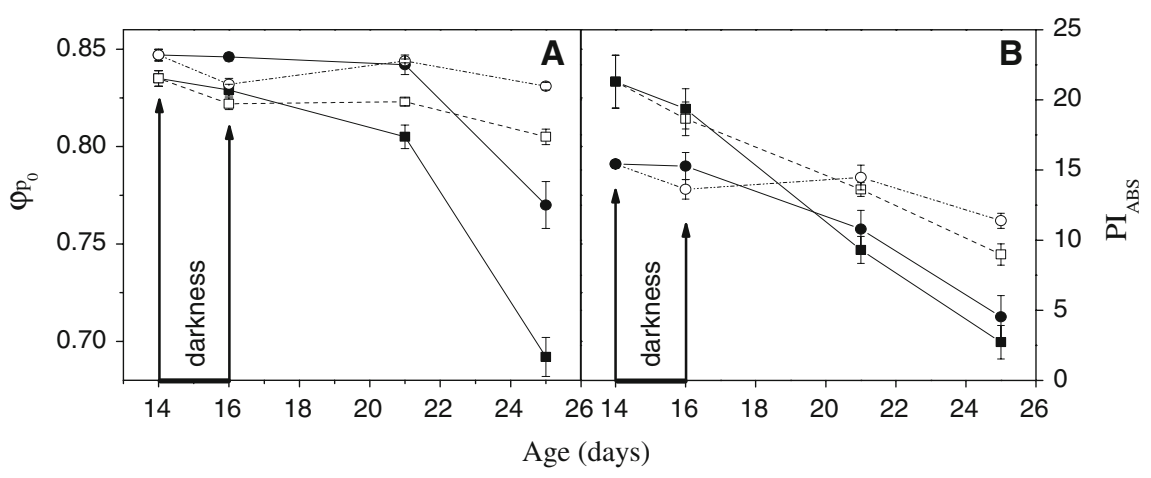

Fig. 2 Time-course changes in the maximum quantum yield of PSII photochemistry $\varphi_{\mathrm{P}_{0}}$ (a) and the performance index PI (b) in control cotyledons ( - ) and primary leaves $(-\bullet)$, as well as in cotyledons ( $\square-)$ and leaves ( $-\circ$ ) of whole plants

primary photochemistry as well as the electron transport processes, thus making it more sensitive for assessment of senescence acceleration (Strasser et al. 2004). Two-day darkening of whole plants did not change significantly both the $F_{\mathrm{V}} / F_{\mathrm{M}}$ ratio and PI neither in the cotyledons nor in the primary leaves (Fig. 2a, b). However, the values for both parameters remained higher as compared to the respective controls during the 9-day recovery period. In the cotyledons upon reillumination PI continued to decrease whereas it remained relatively constant in the primary leaves (Fig. 2b).

Concerning $\psi_{0}$ which describes the efficiency of $Q_{\mathrm{A}}{ }^{-}$reoxidation, our results showed a gradual decrease till day 25 in both leaf organs (Fig. 3). No significant differences in this parameter were detected in the cotyledons and leaves after 2-day dark treatment. A gradual decrease was observed during the recovery period, the values staying higher compared with the controls (Fig. 3).

The effective dissipation of untrapped excitation energy from the active reaction centers $\left(\mathrm{DI}_{0} / \mathrm{RC}\right)$ showed a sharp increase after day 21 in both leaf organs, the values measured in the yellow 25-day-old cotyledons being higher as compared with the leaves (Fig. 4). The effective dissipation in both cotyledons and leaves following 2-day dark treatment was slightly increased and it remained relatively low and constant during the 9-day recovery period.

A similar decrease in the dynamics of the quantum yield of electron transport from $Q_{\mathrm{A}}{ }^{-}$to the PSI end electron acceptors $\left(\varphi_{\mathrm{R}_{0}}\right)$ in the cotyledons and leaves was measured with progression of natural senescence darkened for 2 days and recovered for 9 days. Measurements were done with attached leaf organs and represent the means of 15 repetitions from three independent experiments

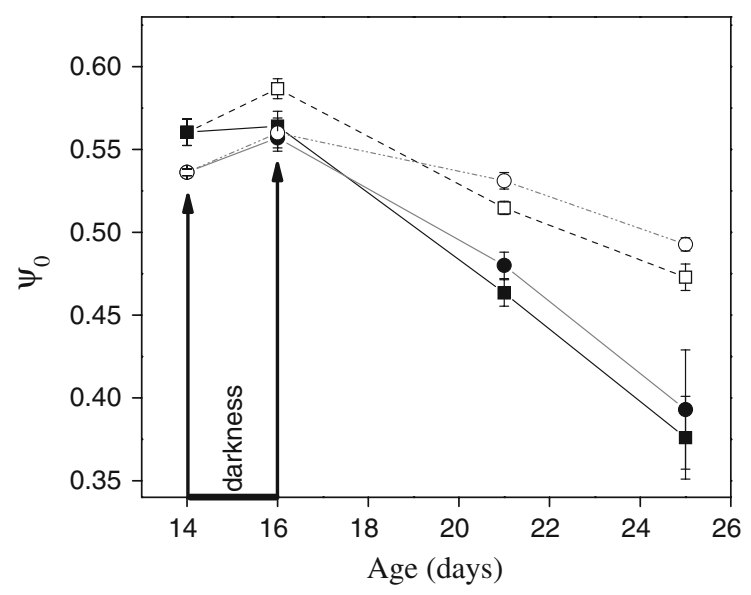

Fig. 3 Time-course changes in the efficiency for conversion of excitation energy flux to electron transport flux $\left(\psi_{0}\right)$ in: control (-), darkened for 2 days and recovered for 9 days cotyledons $(-\square-)$ as well as control $(\multimap-)$, darkened for 2 days and recovered for 9 days primary leaves ( --$)$ ) of zucchini plants. According to the JIP-test $\psi_{0}$ is the probability (at $t=0$ ) that a trapped exciton moves an electron into the electron transport chain beyond $Q_{\mathrm{A}}{ }^{-}$. Measurements were done with attached leaf organs and represent the means of 15 repetitions from three independent experiments

(Fig. 5a, b). However, the response of the differentiated leaves to the applied dark stress was apparently different from that of the cotyledons. An inhibition by $30 \%$ was registered in the leaves after 2-day dark treatment (Fig. 5b). By contrast, in the cotyledons, $\varphi_{\mathrm{R}_{0}}$ remained unchanged after dark treatment followed by a gradual decrease, the values remaining higher compared with the control. In the leaves, upon re-illumination $\varphi_{\mathrm{R}_{0}}$ gradually increased till day 21 


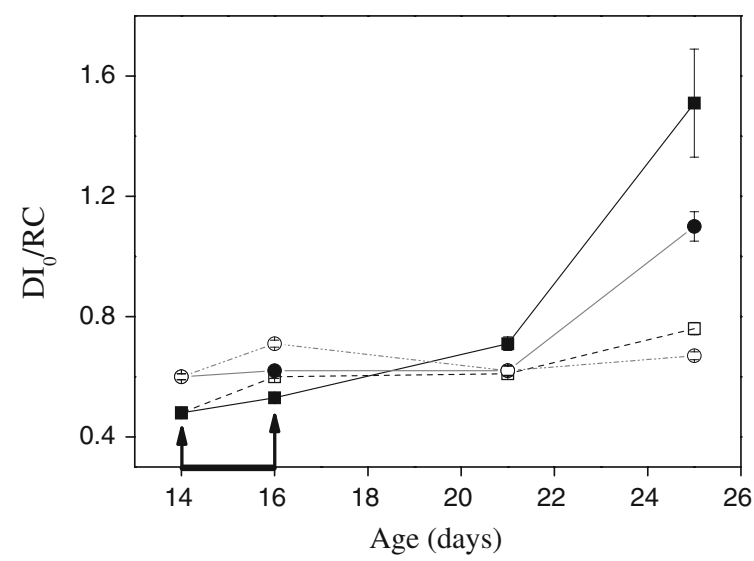

Fig. 4 Time-course changes in the effective dissipation of an active PSII reaction center $\left(\mathrm{DI}_{0} / \mathrm{RC}\right)$ at $t=0$ in: control (--), darkened for 2 days and recovered for 9 days cotyledons $(\neg-)$ as well as control $(\bullet-)$, darkened for 2 days and recovered for 9 days primary leaves ( $-\mathrm{O}_{-}$) of zucchini plants. Measurements were done with attached leaf organs and represent the means of 15 repetitions from three independent experiments

followed by a decline till day 25 . Thus, on day 25 the activity of the PSI end electron acceptors in the cotyledons and the primary leaves of plants recovering from the dark stress exceeded that in the yellowing controls by 72 and $79 \%$, respectively.

\section{$\mathrm{P}_{700}$ oxidation}

The effect of darkness on PSI activity in cotyledons and primary leaves was estimated by measuring the far-red induced changes in $\mathrm{P}_{700}$ redox state. A decrease in the $\mathrm{P}_{700}$ oxidation levels was observed in both cotyledons and leaves of control plants with progression of natural senescence (Fig. 6). Two-day dark treatment of the plants did not affect the $\mathrm{P}_{700}$ oxidation levels in the cotyledons. Furthermore, the values for $\mathrm{P}_{700}$ oxidation were similar to the control also during the subsequent recovery period. In contrast to cotyledons, a decrease in the values for $\mathrm{P}_{700}$ oxidation levels by $24 \%$ was registered in the leaves following 2-day dark treatment (Fig. 6). Five days after returning the plants to normal light regime the $\mathrm{P}_{700}$ oxidation levels in the leaves reached the control values. In general, the changes in the $\mathrm{P}_{700}$ oxidation levels showed the same trend as the timecourse changes in Chl content (Fig. 1) in both cotyledons and leaves.

\section{Discussion}

Leaf yellowing is the most convenient visible indicator of leaf senescence which reflects mainly chloroplast senescence of mesophyll cells (Smart 1994; Lim et al. 2007). A decrease in the content of both chlorophylls and carotenoids during natural senescence was observed in zucchini cotyledons and primary leaves (Fig. 1; Table 1). The differences found in total chlorophyll content as well as in the life span between cotyledons and primary leaves can be interpreted in terms of the specific functions of cotyledons, being reserve organs during the early stages of germination which later function as

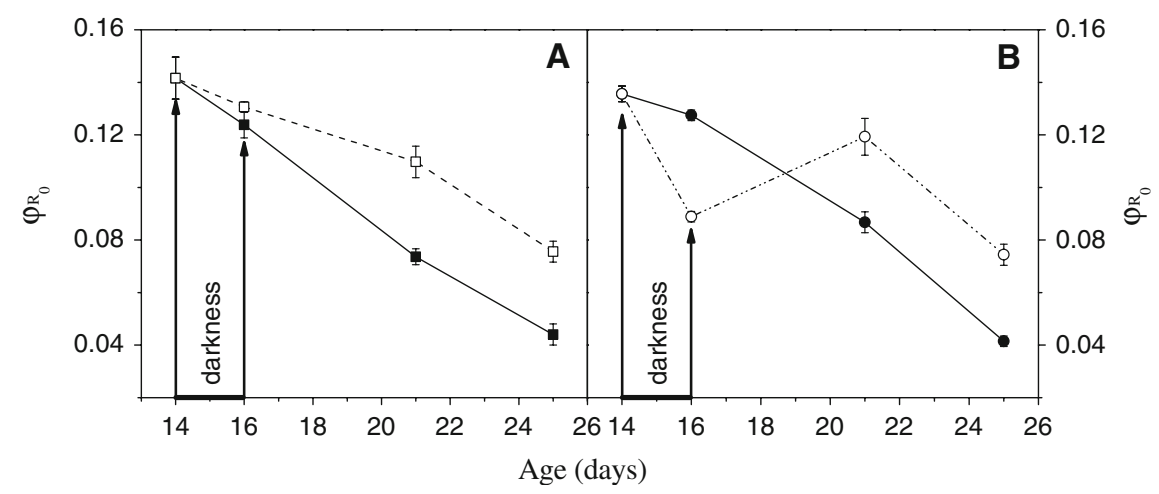

Fig. 5 The quantum yield of electron transport from $Q_{\mathrm{A}}{ }^{-}$to PSI end electron transport acceptors $\left(\varphi_{\mathrm{R}_{0}}\right)$ in: a control (- - ), darkened for 2 days and recovered for 9 days cotyledons $(\multimap-)$; b control (־), darkened for 2 days and recovered for 9 days primary leaves (-○-) of zucchini plants. Measurements were done with attached leaf organs and represent the means of 15 repetitions from three independent experiments 


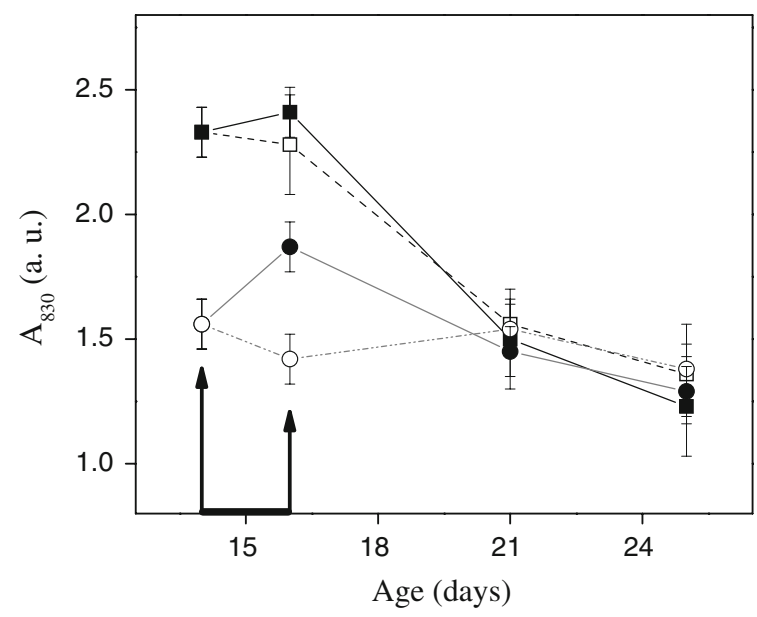

Fig. 6 Time-course changes in the far-red induced $\mathrm{P}_{700}$ oxidation levels in: control ( --$)$, darkened for 2 days and recovered for 9 days cotyledons $(-\square)$ as well as control $(--)$, darkened for 2 days and recovered for 9 days primary leaves (- - ) of zucchini plants. The $\mathrm{A}_{830}$ signal was determined after oxidizing $\mathrm{P}_{700}$ by irradiation with far-red light $\left(13.4 \mathrm{~W} \mathrm{~m}^{-2}\right)$

photosynthesizing organs (La Rocca et al. 1996; Kutik et al. 1998; Ananieva et al. 2008a). The timecourse changes in total chlorophyll content in zucchini cotyledons were in accordance with the progressive decline in the photosynthetic rate reported recently (Ananieva et al. 2008a), thus ensuring their longer life span and slower senescence as compared to primary leaves. Moreover, the time of cotyledon death coincided with the formation of the 9th-10th differentiated leaves and the beginning of flowering, thus confirming the correlative control of their senescence (Lim et al. 2007).

In order to eliminate the effect of age as an additional strong inducer of natural senescence, we used relatively young cotyledons and primary leaves. As we aimed to investigate simultaneously the effect of darkness on both cotyledons and leaves, we used 14day-old plants. Our recent results showed that the amount of reserve globulins in the cotyledons at that growth stage was significantly reduced (Ananieva et al. 2008a), thus indicating that the mixotrophic metabolism typical for the early stages of cotyledon development had been changed to fully autotrophic characteristic for the true leaves (Kutik et al. 1998). Thus, although cotyledons and leaves were not of the same age, they were characterized with similar physiological status. Our results showed that the transfer of whole plants to darkness for 2 days caused a reduction in the pigment content only in the primary leaves whereas total chlorophyll in the cotyledons remained unchanged (Fig. 1). Moreover, when dark treatment was applied at the age of 8 days when cotyledon chlorophyll accumulation reached its maximum, again no significant changes were registered in total chlorophyll amount (data not shown). These results indicate that primary leaves were more susceptible to the dark stress compared with cotyledons.

It has been established that during dark-induced senescence the $\mathrm{Chl} a / b$ ratio remains constant whereas under developmental senescence a decline of this ratio is observed (Krupinska and Humbeck 2004; Keech et al. 2007). The latter is due to an enhanced stability of the light-harvesting complexes containing $\mathrm{Chl} b$ in comparison to the reaction center complexes including exclusively Chl $a$ (Humbeck et al. 1996; Miersch et al. 2000). In agreement with the above results, we showed a stronger decrease in Chl $a$ content as compared to $\mathrm{Chl} b$ in both cotyledons and primary leaves during natural senescence, thus leading to a reduction in the $\mathrm{Chl} a / b$ ratio (Table 1). In contrast, 2-day dark treatment did not affect the $\mathrm{Chl} a / b$ ratio in both leaf organs, due to a similar reduction in Chl $a$ and $\mathrm{Chl} b$ in the primary leaves whereas in the cotyledons both $\mathrm{Chl} a$ and Chl $b$ remained unchanged (Table 1). Likewise the timecourse changes in total chlorophyll content, the results on the $\mathrm{Chl} a / b$ ratio in both leaf organs confirm again the higher resistance of cotyledons to the applied dark stress as compared to the primary leaves.

After a 9-day recovery period total $\mathrm{Chl}$ and Car contents in both cotyledons and primary leaves were higher than in controls (Fig. 1; Table 1), thus indicating delayed senescence. However, in Arabidopsis the senescence of cotyledons was not reversed by the return to light, but rather accelerated (Weaver and Amasino 2001). Except for the already mentioned different senescence character in Arabidopsis (Lim et al. 2007), this discrepancy could be also due to the different light regime. In the case of accelerated senescence following dark treatment, Arabidopsis plants were grown under continuous light which normally led to shortened vegetative phase whereas in our study, zucchini plants were grown under a light/dark cycle which eventually resulted in delayed senescence during the recovery period. 
The photochemical quantum efficiency of PSII is characterized by the $F_{\mathrm{V}} / F_{\mathrm{M}}$ ratio representing the efficiency of energy capture by open PSII centers and reflecting the changes in the structural organization of PSA (Maxwell and Johnson 2000). The $F_{\mathrm{V}} / F_{\mathrm{M}}$ ratio is highly conserved and decreases with progression of senescence or due to different stress factors (Hunt 2003; Gan 2004). Our results showed that the $F_{\mathrm{V}} / F_{\mathrm{M}}$ ratio $\left(\varphi_{\mathrm{P}_{0}}\right.$ in the JIP-test) in both cotyledons and leaves was affected only at later stages of natural senescence (Fig. 2a) which could be accounted for by the drastic decline in pigment content. A similar result was observed previously in senescing barley leaves where the $F_{\mathrm{V}} / F_{\mathrm{M}}$ ratio decreased only at a later point of time (Humbeck et al. 1996; Miersch et al. 2000).

In yellow senescing cotyledons a strong inhibition in the functional activity of PSA based on performance index (PI) of PSII was also observed (Fig. 2b). The PI values measured in control primary leaves on day 25 were close to those of the yellow cotyledons but the $F_{\mathrm{V}} / F_{\mathrm{M}}$ ratio remained relatively high (Fig. 2a, b) which could be accounted for by the different age of cotyledons and primary leaves due to the earlier appearance of cotyledons in ontogenesis (day 4) as compared to primary leaves (days 7-8) (Mishev et al. 2005).

Two-day dark treatment did not cause any significant damage in the structure and functional activity of PSA (Fig. 2a, b), thus indicating that darkening of whole plants did not induce senescence in both leaf organs. Similar lack of inhibition in the maximal efficiency of PSII photochemistry together with a decrease in chlorophyll content was also found in senescing wheat leaves within the first 3 days of whole-plant dark treatment ( $\mathrm{Lu}$ and Zhang 1998). Moreover, only a slight decrease in the $F_{\mathrm{V}} / F_{\mathrm{M}}$ ratio in Arabidopsis leaves after 6-day darkening of whole plants has been recently reported which contrasted with the rapid decline in individually darkened leaves after the second day of dark treatment (Keech et al. 2007).

It must be noted that in the cotyledons $\varphi_{\mathrm{P}_{0}}$ remained constant during the 9-day recovery period following dark stress whereas PSII functional activity as revealed by PI was further decreasing (Fig. 2a, b). In the primary leaves not only $\varphi_{\mathrm{P}_{0}}$ (Fig. 2a), but also PI (Fig. 2b) remained unchanged, thus implying preserved both structure and functional activity of
PSII. A similar effect of senescence repression due to whole-plant dark treatment was demonstrated earlier for Arabidopsis true leaves only, but not for cotyledons (Weaver and Amasino 2001). Our results on cotyledons are in accordance with the recently proposed idea for DP leaves whose metabolism enters a "stand-by mode" in order to preserve the capacity of photosynthetic machinery (Keech et al. 2007).

The gradual decline of PI along with the preservation of relatively constant values for $\varphi_{\mathrm{P}_{0}}$ in the course of senescence suggested the existence of specific regulatory mechanisms that determine the down-regulation of PSII functioning. In order to study in more details the effect of short-term dark treatment on specific components of the electron transport chain we used additional parameters derived from the JIP-test as well as the dynamics in the farred induced $\mathrm{P}_{700}$ oxidation levels. A progressive decrease in the efficiency of $Q_{\mathrm{A}}{ }^{-}$reoxidation $\left(\psi_{0}\right)$ was registered during natural senescence in both leaf organs (Fig. 3). At later stages of ontogenesis it was accompanied by stimulation in the effective dissipation of untrapped excitation energy from the active PSII reaction centers $\left(\mathrm{DI}_{0} / \mathrm{RC}\right)$ reflecting the massive destruction of LHCs (Fig. 4). Recent experiments with bean leaves revealed a similar down-regulation of PSII activity during natural senescence due to PSII reaction centers inactivation which preceded the degradation of antenna complexes (Yordanov et al. 2008).

The transfer of whole plants to darkness did not induce any significant changes in $\psi_{0}$ and $\mathrm{DI}_{0} / \mathrm{RC}$ in both cotyledons and leaves (Figs. 3, 4). During the recovery period after reillumination a higher number of active PSII reaction centers was found when compared with the controls (Fig. 3). Furthermore, the rate of dissipation was also maintained at a constant level in the recovering cotyledons and primary leaves with senescence progression (Fig. 4). Thus, the delayed loss of photosynthetic pigments during the recovery period (Fig. 1) was in accordance with retarded disorganization of the antenna complexes and lower rate of dissipation in both leaf organs. These results indicated again that senescence was delayed in cotyledons and primary leaves due to whole-plant dark treatment.

The effect of the applied dark treatment on $\mathrm{P}_{700}$ activity was also studied. The PSI activity was 
estimated directly by the changes in the $\Delta \mathrm{A}_{830}$ leaf absorbance as well as by the recently introduced JIP-test parameter $\varphi_{\mathrm{R}_{0}}$ representing the quantum yield of electron transport from $Q_{\mathrm{A}}^{-}$to the PSI end electron acceptors (Tsimilli-Michael and Strasser 2008; Smit et al. 2008). Recently, a correlation between the changes in $\Delta \mathrm{A}_{830}$ leaf absorbance and $\varphi_{\mathrm{R}_{0}}$ in decapitated and defoliated bean plants was found (Yordanov et al. 2008). In the present study, these two different experimental approaches also demonstrated a good correlation (Figs. 5, 6). In contrast to PSII, darkness lowered PSI activity as judged by the two parameters only in the leaves. The contribution of PSI cyclic electron transport as the reason for decreased $\Delta \mathrm{A}_{830}$ can be excluded because of the similarity in the decay kinetics between dark-treated and control leaves after switching off the far-red light (data not shown). As judged by $\varphi_{\mathrm{R}_{0}}$, the lower $\mathrm{P}_{700}$ oxidation levels in the darktreated leaves could be associated with a rise in PSI reaction center recombinations as a result of reduced electron transport activity in the PSI acceptor side (Schansker et al. 2005). Moreover, a close correlation between the changes in $\mathrm{Chl}$ content and the $\Delta \mathrm{A}_{830}$ signal in the primary leaves was observed, thus indicating that the changes in $\mathrm{P}_{700}$ activity were determined to a greater extent by the changes in pigment content.

\section{Conclusion}

In this report we demonstrate different responses of cotyledons and true leaves of C. pepo (zucchini) to whole-plant dark treatment. Our results based on chlorophyll content, PSII and PSI activities showed delayed senescence in cotyledons in contrast to Arabidopsis cotyledons where senescence was accelerated but not delayed during the recovery period after whole-plant dark treatment (Weaver and Amasino 2001), thus implying the existence of different mechanisms of cotyledon senescence. Furthermore, the lack of changes in pigment content as well as in the activities of PSII and PSI showed higher resistance of cotyledons to the applied dark stress as compared to the primary leaves. In addition, PSI activity in the leaves was more susceptible to dark treatment when compared with PSII.
Acknowledgments Assoc. Prof. Zhivko Danailov from the Institute of Genetics, Sofia is thanked for kindly providing the PEA apparatus used throughout the experiments. This work was supported by the National Science Fund at the Bulgarian Ministry of Education and Science (Grant No. MU-B-1518/ 2005).

\section{References}

Ananieva K, Malbeck J, Kamínek M, van Staden J (2004) Changes in endogenous cytokinin levels in cotyledons of Cucurbita pepo L. (zucchini) during natural and darkinduced senescence. Physiol Plant 122:133-142. doi: 10.1111/j.1399-3054.2004.00378.x

Ananieva K, Ananiev ED, Mishev K, Georgieva K, Malbeck J, Kamínek M, van Staden J (2007) Methyl jasmonate is a more effective senescence-promoting factor in Cucurbita pepo (zucchini) cotyledons when compared with darkness at the early stage of senescence. J Plant Physiol 164:11791187. doi:10.1016/j.jplph.2006.07.008

Ananieva K, Ananiev ED, Mishev K, Georgieva K, Tzvetkova N, Van Staden J (2008a) Changes in photosynthetic capacity and polypeptide patterns during natural senescence and rejuvenation of Cucurbita pepo L. (zucchini) cotyledons. Plant Growth Regul 54:23-29. doi:10.1007/ s10725-007-9223-x

Ananieva K, Ananiev ED, Doncheva S, Georgieva K, Tzvetkova N, Kamínek M, Motyka V, Dobrev P, Gajdošová S, Malbeck J (2008b) Senescence progression in a single darkened cotyledon depends on the light status of the other cotyledon in Cucurbita pepo (zucchini) seedlings: potential involvement of cytokinins and cytokinin oxidase/dehydrogenase activity. Physiol Plant 134:609623. doi:10.1111/j.1399-3054.2008.01161.x

Arnon DI (1949) Copper enzymes in isolated chloroplasts. Polyphenoloxidase in Beta vulgaris. Plant Physiol 24: $1-15$

Biswal UC, Biswal B (1984) Photocontrol of leaf senescence. Photochem Photobiol 39:875-879. doi:10.1111/j.17511097.1984.tb08874.x

Gan S (2004) The hormonal regulation of leaf senescence. In: Davies PJ (ed) Plant hormones: biosynthesis signal transduction and action. Kluwer Academic Publishers, Dordrecht, pp 561-581

Ghosh S, Mahoney S, Penterman J, Peirson D, Dumbroff E (2001) Ultrastructural and biochemical changes in chloroplasts during Brassica napus senescence. Plant Physiol Biochem 39:777-784. doi:10.1016/S0981-9428(01)012 96-7

Grover A (1993) How do senescing leaves lose photosynthetic activity? Curr Sci 64:226-234

Humbeck K, Krupinska K (2003) The abundance of minor chlorophyll $a / b$-binding proteins CP29 and LHCI of barley (Hordeum vulgare L.) during leaf senescence is controlled by light. J Exp Bot 54:375-383. doi:10.1093/ jxb/54.381.375

Humbeck K, Quast S, Krupinska K (1996) Functional and molecular changes in the photosynthetic apparatus during senescence of flag leaves from field-grown barley plants. 
Plant Cell Environ 19:337-344. doi:10.1111/j.1365-3040. 1996.tb00256.x

Hunt S (2003) Measurements of photosynthesis and respiration in plants. Physiol Plant 117:314-325. doi:10.1034/j.13993054.2003.00055.x

Keech O, Pesquet E, Ahad A, Askne A, Nordvall D, Vodnala $\mathrm{S}$, Tuominen H, Hurry V, Dizengremel P, Gardestrom P (2007) The different fates of mitochondria and chloroplasts during dark-induced senescence in Arabidopsis leaves. Plant Cell Environ 30:1523-1534. doi:10.1111/ j.1365-3040.2007.01724.X

Kim HJ, Ryu H, Hong SH, Woo HR, Lim PO, Lee IC, Sheen J, Nam HG, Hwang I (2006) Cytokinin-mediated control of leaf longevity by AHK3 through phosphorylation of ARR2 in Arabidopsis. Proc Natl Acad Sci USA 103:814819. doi:10.1073/pnas.0505150103

Klughammer C, Schreiber U (1998) Measuring $\mathrm{P}_{700}$ absorbance changes in the near infrared spectral region with a dual wavelength pulse modulation system. In: Garab G (ed) Photosynthesis: mechanisms and effects, vol 5. Kluwer Academic Publishers, The Netherlands, pp 43574360

Krupinska K, Humbeck K (2004) Photosynthesis and chloroplast breakdown. In: Nooden L. (ed) Plant cell death processes. Academic Press, San Diego, pp 169-187

Kutik J, Wilhelmova N, Snopek J (1998) Changes in ultrastructure of Phaseolus vulgaris L. Photosynthetica 35:361-367. doi:10.1023/A:1006908217443

La Rocca N, Barbato R, Casadoro G, Rascio N (1996) Early degradation of photosynthetic membranes in carob and sunflower cotyledons. Physiol Plant 96:513-518. doi: 10.1111/j.1399-3054.1996.tb00466.x

Lim PO, Kim HJ, Nam HG (2007) Leaf senescence. Annu Rev Plant Biol 58:115-136. doi:10.1146/annurev.arplant. 57.032905 .105316

Lu C, Zhang J (1998) Changes in photosystem II function during senescence of wheat leaves. Physiol Plant 104:239-247. doi:10.1034/j.1399-3054.1998.1040212.x

Maxwell K, Johnson GN (2000) Chlorophyll fluorescence-a practical guide. J Exp Bot 51:659-668. doi:10.1093/ jexbot/51.345.659

Miersch I, Heise J, Zelmer I, Humbeck K (2000) Differential degradation of the photosynthetic apparatus during leaf senescence in barley (Hordeum vulgare L.). Plant Biol 2:618-623. doi:10.1055/s-2000-16632

Mishev K, Todorov I, Ananiev ED (2005) Senescence and rejuvenation in intact cotyledons of Cucurbita pepo $\mathrm{L}$. (zucchini). Gen Appl Plant Physiol 31:15-27

Nooden LD (1988) The phenomena of senescence and aging. In: Nooden LD, Leopold AC (eds) Senescence and aging in plants. Academic Press, London, pp 2-51

Nooden LD, Guiamet JJ, John I (1997) Senescence mechanisms. Physiol Plant 101:746-753. doi:10.1111/j.13993054.1997.tb01059.x

Oh S, Lee S, Chung I, Lee C, Nam H (1996) A senescenceassociated gene of Arabidopsis thaliana is distinctively regulated during natural and artificially induced leaf senescence. Plant Mol Biol 30:739-754. doi:10.1007/ BF00019008
Prakash JSS, Baig MA, Bhagwat AS, Mohanty P (2003) Characterisation of senescence-induced changes in light harvesting complex II and photosystem I complex of thylakoids of Cucumis sativus cotyledons: age induced association of LHCII with photosystem I. J Plant Physiol 160:175-184. doi:10.1078/0176-1617-00529

Schansker G, Toth SZ, Strasser RJ (2005) Methylviologen and dibromothymoquinone treatments of pea leaves reveal the role of photosystem I in the $\mathrm{Chl}$ a fluorescence rise OJIP. Biochim Biophys Acta 1706:250-261. doi:10.1016/ j.bbabio.2004.11.006

Smart CM (1994) Gene expression during leaf senescence. New Phytol 126:419-448. doi:10.1111/j.1469-8137.1994. tb04243.x

Smit M, Kruger G, van Heerden P, Pienaar J, Weissflog L, Strasser RJ (2008) Effect of trifluoroacetate, a persistant degradation product of fluorinated hydrocarbons, on $\mathrm{C} 3$ and C4 crop plants. In: Allen J, Gannt E, Golbeck J, Osmond B (eds) Photosynthesis. Energy from the sun. 14th International congress on photosynthesis. Springer, England, pp 1501-1504

Strasser RJ, Tsimilli-Michael M, Srivastava A (2004) Analysis of the chlorophyll a fluorescence transient. In: Papageorgiou GC, Govindjee A (eds) Chlorophyll $a$ fluorescence: a signature of photosynthesis. Kluwer Academic Publishers, The Netherlands, pp 321-362

Thimann KV (1980) The senescence of leaves. In: Thimann KV (ed) Senescence in plants. CRC press, Boca Raton, pp 85-115

Thomas H, Stoddart JL (1980) Leaf senescence. Annu Rev Plant Physiol 31:83-111. doi:10.1146/annurev.pp.31.060 180.000503

Tsimilli-Michael M, Strasser RJ (2008) In vivo assessment of stress impact on plants' vitality: applications in detecting and evaluating the beneficial role of mycorrhization on host plants. In: Varma A (ed) Mycorrhiza: state of the art, genetics and molecular biology, ecofunction, biotechnology, eco-physiology, structure and systematics, vol 3. Springer, England, pp 679-703

van Staden J, Cook EL, Nooden LD (1988) Cytokinins and senescence. In: Nooden LD, Leopold AC (eds) Senescence and aging in plants. Academic Press, San Diego, pp 281-328

Weaver LM, Amasino RM (2001) Senescence is induced in individually darkened Arabidopsis leaves, but inhibited in whole darkened plants. Plant Physiol 127:876-886. doi: 10.1104/pp.010312

Yamagishi M, Yamamoto Y (1994) Effects of boron on nodule development and symbiotic nitrogen fixation in soybean plants. Soil Sci Plant Nutr 40:265-274

Yordanov I, Goltsev V, Stefanov D, Chernev P, Zaharieva I, Kirova M, Gecheva V, Strasser RJ (2008) Preservation of photosynthetic electron transport from senescenceinduced inactivation in primary leaves after decapitation and defoliation of bean plants. J Plant Physiol 165:19541963. doi:10.1016/j.jplph.2008.05.003 